\title{
Valérie Guyen-Croquez, Tradition et originalité dans les Croniques et Conquestes de Charlemaine de David Aubert
}

\section{Anne Schoysman}

\section{(2) OpenEdition \\ 12 Journals}

\section{Édition électronique}

URL : http://journals.openedition.org/studifrancesi/4314

DOI : 10.4000/studifrancesi.4314

ISSN : 2421-5856

Éditeur

Rosenberg \& Sellier

\section{Édition imprimée}

Date de publication : 1 septembre 2016

Pagination : 309-310

ISSN : 0039-2944

\section{Référence électronique}

Anne Schoysman, «Valérie Guyen-Croquez, Tradition et originalité dans les Croniques et Conquestes de Charlemaine de David Aubert », Studi Francesi [En ligne], 179 (LX | II) | 2016, mis en ligne le 01 septembre 2016, consulté le 18 septembre 2020. URL : http://journals.openedition.org/studifrancesi/4314 ; DOI : https://doi.org/10.4000/studifrancesi.4314

Ce document a été généré automatiquement le 18 septembre 2020.

\section{cc) (†) $\odot$}

Studi Francesi è distribuita con Licenza Creative Commons Attribuzione - Non commerciale - Non opere derivate 4.0 Internazionale. 


\title{
Valérie Guyen-Croquez, Tradition et originalité dans les Croniques et Conquestes de Charlemaine de David Aubert
}

\author{
Anne Schoysman
}

\section{RÉFÉRENCE}

VALÉRIE GUYEN-CROQUEZ, Tradition et originalité dans les Croniques et Conquestes de Charlemaine de David Aubert, Paris, Honoré Champion, 2015, «Bibliothèque du $\mathrm{xv}^{\mathrm{e}}$ siècle» $79,466 \mathrm{pp}$.

1 Les Croniques et Conquestes de Charlemaine [CCC], signées au colophon par David Aubert en 1458, sont conservées dans trois luxueux volumes (Bruxelles, KBR, mss 9066 à 9068 , illustrés par Jean le Tavernier) et dédiées à Philippe le Bon (à Jean de Créquy à l'origine, dans le vol. 1). À l'exemple de ceux qui «font nouvelles transcriptions et mutation de rime en prose, en termes si bien couchiés qu'il semble que rhetorique propre leur mette ou cueur ce qu'ilz escripvent» (Prologue au livre I, éd. Guiette, I, Bruxelles, 1940, p. 13), David Aubert réalise une vaste compilation originale sur la base de chroniques et épopées nombreuses et difficiles à débrouiller: le Pseudo-Turpin traduit par Jean de Boulogne, les Grandes Chroniques de France, Doon de Maience, Girart de Vienne, Ogier, la Chanson de Roland, Aspremont, Guillaume d'Orange, la Chanson des Saisnes de Jean Bodel, Fierabras et d'autres encore. Si le contexte historique et l'étude des sources ont déjà été éclairés au mieux (cf. B. Bousmanne et G. Palumbo, La librairie des ducs de Bourgogne, IV, Turnhout, Brepols, 2009, pp.127-158), une analyse structurelle et stylistique approfondie du texte restait à faire. Elle est d'autant plus intéressante qu'elle s'impose si l'on veut essayer de préciser le rôle mal défini de David Aubert, «escripvain» et «clerc», soit à la fois historien penché sur les sources, compilateur, metteur en prose, 
remanieur-auteur, copiste-éditeur; aussi la question difficile que pose d'emblée Valérie Guyen-Croquez est-elle celle de la «part de création de l'escripvain» (p. 16).

2 L'étude comprend cinq parties. Après une «Présentation générale» des données biographiques et historiques sur David Aubert et sur les commanditaires du texte, sur la cour de Bourgogne, ainsi qu'un résumé des $C C C$, la deuxième partie («Des sources nombreuses au service d'un créateur original», pp.45-115) fait le point sur l'émancipation de David Aubert vis-à-vis de ses sources, en vers mais aussi souvent réécritures récentes en prose. Il travaille en esquissant une trame générale à partir du Pseudo-Turpin ou des Grandes Chroniques de France, où il insère des éléments glanés dans les textes épiques, découpés et recomposés. L'histoire de Charlemagne permettant la fusion de la chronique et de l'épique (conquestes) dans un ensemble homogène, Aubert «met à profit deux traditions sans relever d'aucune des deux» (p. 75).

3 Suit une troisième partie («Le risque de l'hétérogénéité», pp.117-188) consacrée à l'étude de la structure du texte, de ses procédés narratifs, du traitement de l'espace et $\mathrm{du}$ temps. Dans la macrostructure, sutures et transitions témoignent d'une «conjointure entre les grands épisodes qui joue sur la surprise du découpage, le rappel des événements, les thématiques identiques, les tonalités» (p.125), et dans la microstructure narrative, les parallélismes, juxtapositions, amplifications des scènes épiques stéréotypées concourent au même effet de cohérence (pp.153-164). Il est intéressant de noter, par exemple, que les tables des matières élaborées par Aubert constituent une synthèse originale et ne se limitent pas à reprendre les rubriques de chapitres, dont l'analyse (pp.132-152) révèle par ailleurs un rôle structurant plutôt qu'une fonction descriptive, comme c'est par contre le cas dans d'autres proses dérivées d'une source unique (p. 140).

4 La quatrième partie («Une écriture sous tension: répétition et invention», pp. 189-286), consacrée à l'étude de la langue, n'est pas conçue pour satisfaire aux exigences de l'historien de la langue; la bibliographie de référence se limite du reste aux outils lexicographiques et grammaticaux les plus courants. Mais la perspective de cette étude, visant à montrer l'originalité de l'élaboration d'un récit doté d'une cohérence propre, justifie l'approche plus proprement rhétorique et stylistique qui est choisie ici pour l'examen de la syntaxe, des procédés emphatiques et métaphoriques, du lexique, du discours direct, de la valeur épique des expressions proverbiales, des portraits brossés par Aubert. De nombreux exemples montrent que le narrateur recourt généralement à des métaphores traditionnelles, à un lexique archaïsant, à un usage exemplificatif plutôt que lyrique des proverbes, marques de distanciation qui contrastent avec une maitrise remarquable et toute personnelle du discours direct (pp. 249-258). Alors que la narration épique est fondée sur la juxtaposition, comme ses contemporains Aubert «préfère l'explication à la juxtaposition» (p. 400), et «c'est surtout dans la description du processus qui conduit du sentiment à l'action qu'il est original» (p. 185).

5 La dernière partie s'intitule «Thèmes et personnages. L'inspiration épique: souffle ou soupir?» (pp. 287-398). Les combats, l'idéal de la croisade, les manifestations de la dévotion, le traitement de l'altérité, de la féodalité s'affaiblissant, les valeurs de substitution que sont l'idéal courtois et le rôle du féminin, le comique, les valeurs nouvelles qui s'expriment par la mélancolie, le silence ou le regard porté sur le monde: V. G.-C. exploite toutes les ressources du texte pour sonder l'originalité de David Aubert face à la tradition. Nombreuses sont les suggestions que l'on tire des ces pages, notamment à propos du statut du lecteur: «Le texte évoque donc un monde en 
mutation où certaines valeurs guerrières ont évolué; l'idée de croisade semble se retirer du texte, la religion reste présente mais subit des modifications. Face à ces déplacements, le lecteur peut s'interroger sur son identité propre, sur ce qui l'unit au narrateur, au personnage du texte» (p. 327). Tout au long de cette étude consacrée aux techniques dont se sert David Aubert pour refondre en un tout cohérent et original la chronique et l'histoire, l'épique et le romanesque, on remarquera la constance avec laquelle réapparaît cet aspect: «Les subtilités de ce texte [les $C C C]$ résident dans le jeu avec l'intertextualité qui sollicite la complicité du lecteur» (p. 405). C'est là une des meilleures preuves de la volonté d'élaborer un texte original et de le soumettre au jugement d'un lecteur qui «prend une part active à la création du livre: à lui de le corriger, de le compléter» (p. 115).

6 Une bibliographie, un relevé des proverbes et expressions proverbiales (très large, sans référence systématique aux relevés existants) et un index des noms propres clôturent cette étude, dont l'intérêt ne se limite pas aux $C C C$, mais concerne toute l'activité de réécriture en prose à la cour de Bourgogne au milieu du $\mathrm{Xv}^{\mathrm{e}}$ siecle.

7 Signalons qu'il existe un remaniement assez profond du texte (ms. Dresde, LB, O-81, après 1474 , dédié à Philippe de Hornes, † 1488), dont V. G.-C. ne s'occupe pas ici. L'auteur anonyme y déclare (cf. B. Guidot, Croniques et Conquestes de Charlemagne, in Nouveau Répertoire de mises en prose, dir. M. Colombo Timelli, B. Ferrari, A. Schoysman, Fr. Suard, Paris, Classiques Garnier, 2014, p. 156) avoir tiré sa matière d'un ouvrage écrit pour Jean de Créquy, le dédicataire original dont le nom est resté au prologue du premier volume de Bruxelles, et semble donc avoir travaillé à partir d'une version du texte antécédente à celle de l'exemplaire en trois volumes finalement dédicacé à Philippe le Bon en 1458, qui nous est conservé. Il serait utile d'examiner de près la version de Dresde afin de vérifier s'il est possible de déterminer dans quelle mesure elle reflète un état du texte antérieur à la version des mss KBR 9066-9068. 